\title{
Two Centuries of Observing R Coronæ Borealis
}

\author{
Geoffrey C. Clayton
}

Dept. of Physics \& Astronomy, Louisiana State University, Baton Rouge, LA 70803, USA email: gclayton@fenway.phys.lsu.edu

\begin{abstract}
R Coronæ Borealis was found to be variable in the year 1783, and was one of the first variable stars to be so identified. Its class, the R Coronæ Borealis (RCB) stars, are rare hydrogen-deficient carbon-rich supergiants. RCB stars undergo massive declines of up to 8 mag due to the formation of carbon dust at irregular intervals. The mechanism of dust formation around RCB stars is not well understood, but the dust is thought to form in or near the atmosphere of the star. Their rarity may stem from the fact that they are in an extremely rapid phase of the evolution, or are in an evolutionary phase that most stars do not undergo. Several evolutionary models have been suggested to account for the RCB stars, including a merger of two white dwarfs (WDs) or a final helium-shell flash (FF) in a PN central star. The large overabundance of ${ }^{18} \mathrm{O}$ found in most of the RCB stars favours the WD merger model, while the presence of $\mathrm{Li}$ in the atmospheres of five $\mathrm{RCB}$ stars favours the $\mathrm{FF}$ one. In particular, the measured isotopic abundances imply that many, if not most, RCB stars are produced by WD mergers, which may be the low-mass counterparts of the more massive mergers thought to produce type Ia supernovæ. Understanding these enigmatic stars depends to a large extent on continuous monitoring to catch their irregular but rapid variations caused by dust formation, their variations due to stellar pulsations, and long-term changes that may occur over centuries. I will use observations of R Coronæ Borealis obtained over 200 years to demonstrate what kinds of monitoring are necessary for these and similar classes of variables.
\end{abstract}

Keywords. circumstellar matter, dust, evolution, surveys

\section{Introduction}

$\mathrm{R}$ Coronæ Borealis ( $\mathrm{R} \mathrm{CrB}$ ) was one of the first variable stars identified. Its brightness variations have been monitored since its discovery over 200 years ago (Pigott \& Englefield 1797) - see Fig. 1. Clayton et al. (2005, 2007) made the remarkable discovery that cool $\mathrm{R}$ Coronæ Borealis (RCB) stars with $\mathrm{CO}$ bands have ${ }^{18} \mathrm{O} /{ }^{16} \mathrm{O}$ ratios that are orders of magnitude higher than those seen for any other star. The RCB stars are a small group of hydrogen-deficient, carbon-rich supergiants. About 65 are known in the Galaxy and the Magellanic Clouds (Tisserand et al. 2008; Kraemer et al. 2005; Zaniewski et al. 2005; Alcock et al. 2001; Clayton 1996). Their defining characteristics are hydrogen deficiency and unusual variability-RCB stars undergo massive declines of up to 8 mag caused by the formation of carbon dust at irregular intervals. Two models have been proposed for the origin of an RCB star: the Double Degenerate (DD) and the final helium-shell flash (FF) models (Iben et al. 1996; Saio \& Jeffery 2002). The former involves the merger of a CO- and a He-WD (Webbink 1984). In the latter, a star evolving from a planetary nebula (PN) central star expands to supergiant size by a FF (Fujimoto 1977; Renzini 1979). Three stars (Sakurai's Object, V605 Aql and FG Sge) have been observed to undergo FF outbursts that transformed them from hot evolved PN central stars into cool giants with spectral properties similar to RCB stars (Clayton et al. 2006; Asplund et al. 1998, 1999, 2000; Clayton \& De Marco 1997; Gonzalez et al. 1998). 

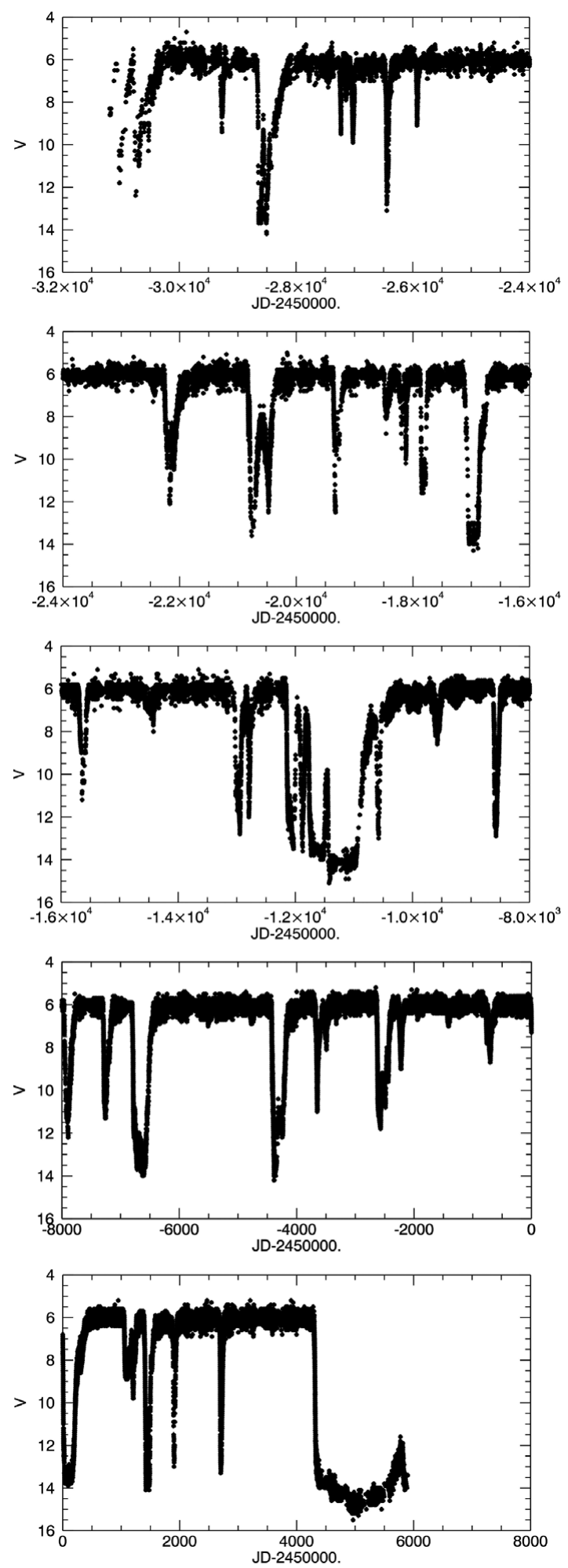

Figure 1. A 100-year light curve for R CrB. Each panel contains 8,000 days. The data plotted are visual AAVSO observations. 
However, Sakurai's Object shows no evidence of ${ }^{18} \mathrm{O}$ (Geballe et al. 2002). Therefore, the FF stars on the one hand, and most of the RCB stars on the other, are likely to be stars with different origins. But the actual observations are somewhat contradictory. Indeed, five RCB stars, including R CrB itself, exhibit enhanced Li abundances, as does the FF star Sakurai's Object (Lambert 1986; Asplund et al. 2000). As shown by Herwig \& Langer (2001), Li enhancements are consistent with the FF model. However, the production of ${ }^{18} \mathrm{O}$ requires temperatures large enough that would destroy completely any Li present. For that reason the simultaneous enrichment of $\mathrm{Li}$ and ${ }^{18} \mathrm{O}$ is not expected in the DD merger model. Unfortunately, all five of those stars are too hot to show $\mathrm{CO}$, so their abundances of ${ }^{18} \mathrm{O}$ are unknown. The presence of $\mathrm{Li}$ in some RCB stars, and also in Sakurai's object, is extremely difficult to explain by a DD merger. Since ${ }^{18} \mathrm{O}$ strongly supports the DD merger/accretion model for most of the stars studied so far, the obvious conclusion is that there are (at least) two evolutionary channels for formation for the RCB stars, the DD perhaps being the dominant mechanism.

\section{Understanding the RCB Stars}

The presence or absence of circumstellar material and the morphology of this material provides a fossil record of previous evolutionary stages. Mass loss occurs in both models during the common-envelope or PN-ejection phases, but the observational manifestations will differ. In the DD model no fossil envelope should remain from the common-envelope phase when the two WDs finally merge to form an RCB star. About $10 \%$ of single stars will undergo a final-flash event (Iben et al. 1996), and about the same percentage of RCB stars (R CrB, RY Sgr, V CrA, and UW Cen) show evidence of resolved fossil dust shells in the far-IR (Walker 1994). At the present time, such a shell has been imaged in the visible around only two RCB stars: UW Cen and R CrB. The shells may be a fossil PN, now neutral, and detectable only in scattered light from dust in the shell. The sizes of the shells are consistent with a PN ejection several thousand years before the FF (Clayton et al. 1999, 2011). UW Cen and R CrB are two of the five RCB stars that show enriched $\mathrm{Li}$, indicating that they may be the product of a FF rather than a DD merger. When in a deep decline, an RCB star can be imaged by using its own dust cloud as a natural coronograph: if it is surrounded by a neutral circumstellar shell, it will be detectable. By applying that technique we can start to get a better statistical sample of RCB stars in order to determine what fraction of them are the result of the FF rather than the DD process.

But RCB stars are very rare. Only about 55 have been discovered in the Galaxy so far (Clayton 1996; Alcock et al. 2001; Zaniewski et al. 2005; Tisserand et al. 2008). The rate of He- and CO-WD mergers is estimated to be about one per century, so if the typical lifetime as an RCB star were about $10^{5}$ years then there would be $\sim 10^{3}$ in the Galaxy. That agrees with the extrapolation to the Galaxy from the number of RCB stars known in the LMC, where a better census can be made. So where are all the RCB stars? The RCB stars may be an 'old bulge' population and there may be $\sim 250$ RCB stars in the reddened 'exclusion' zone toward the bulge (Zaniewski et al. 2005).

Constraints on the spatial distribution and the formation rate of such stars are needed to understand their origins and to test them in the context of actual population-synthesis results. To do so, it is crucial to increase significantly the number of known RCBs. New sky surveys can be used to discover new RCB stars and thus increase the observed sample. Already the ASAS-3 survey has been used to find about 10 new RCB stars. Other surveys coming on line such as CRTS are also being used to look for new RCB stars. 
It is hoped that the results of these surveys will help us distinguish between the FF and DD processes for the formation of RCB stars, and to understand better their population and lifetimes.

\section{References}

Alcock, C., et al. 2001, ApJ, 554, 298.

Asplund, M., Gustafsson, B., Kameswara Rao, N., \& Lambert, D. L. 1998, A\& A, 332, 651.

Asplund, M., et al. 1999, A\&A, 343, 507.

Asplund, M., et al. 2000, A\&A, 353, 287.

Clayton, G. C. 1996, PASP, 108, 225.

Clayton, G. C. \& De Marco, O. 1997, AJ, 114, 2679.

Clayton, G. C., et al. 1999, ApJ, 517, L143.

Clayton, G. C., et al. 2005, ApJ, 623, L141.

Clayton, G. C., et al. 2006, ApJ, 646, L69.

Clayton, G. C., Geballe, T. R., Herwig, F., Fryer, C., \& Asplund, M. 2007, ApJ, 662, 1220.

Clayton, G. C., et al. 2011, ApJ, 743, 44.

Fujimoto, M. Y. 1977, PASJ, 29, 331.

Geballe, T. R., Evans, A., Smalley, B., Tyne, V H., \& Eyres, S. P. S. 2002, Ap\&SS, 279, 39.

Gonzalez, G., et al. 1998, ApJS, 114, 133.

Herwig, F. \& Langer, N. 2001, Nuclear Physics A, 688, 221.

Iben, I., Tutukov, A. V., \& Yungelson, L. R. 1996, ApJ, 456, 750.

Kraemer, K. E., Sloan, G. C., Wood, P. R., Price, S. D., \& Egan, M. P. 2005, ApJ, 631, L147.

Lambert, D. L. 1986, in: K. Hunger, D. Schönberner \& N. Kameswara Rao (eds.), Hydrogen Deficient Stars and Related Objects, IAU Colloq. 87, ASSL, Vol. 128, p. 127

Pigott, E. \& Englefield, H. C. 1797, Phil. Trans. Royal Soc., 87, 33.

Renzini, A. 1979, in: B. E. Westerlund (ed.), Stars and Star Systems, ASSL Vol. 75, p. 155.

Saio, H. \& Jeffery, C. S. 2002, MNRAS, 333, 121.

Tisserand, P., et al. 2008, A\&AA, 481, 673.

Walker, H., 1994, CCP7 Newsletter, 21, 40.

Webbink, R. F., 1984, ApJ, 277, 355.

Zaniewski, A., et al. 2005, AJ, 130, 2293. 